\title{
Seasonal Pattern in the Diagnosis of Gestational Diabetes Mellitus in Southern Sweden
}

\author{
Anastasia Katsarou, ${ }^{1,2}$ Rickard Claesson, ${ }^{1,3}$ Claes Ignell, ${ }^{1,4}$ \\ Nael Shaat, ${ }^{1,2}$ and Kerstin Berntorp ${ }^{1,2}$ \\ ${ }^{1}$ Department of Clinical Sciences, Lund University, Malmö, Sweden \\ ${ }^{2}$ Department of Endocrinology, Skåne University Hospital, Malmö, Sweden \\ ${ }^{3}$ Department of Obstetrics and Gynaecology, Office for Healthcare "Kryh", Ystad, Sweden \\ ${ }^{4}$ Department of Obstetrics and Gynaecology, Office for Healthcare "Sund", Helsingborg, Sweden \\ Correspondence should be addressed to Anastasia Katsarou; anastasia.katsarou@med.lu.se
}

Received 28 October 2016; Accepted 5 December 2016

Academic Editor: Ulrike Rothe

Copyright (C) 2016 Anastasia Katsarou et al. This is an open access article distributed under the Creative Commons Attribution License, which permits unrestricted use, distribution, and reproduction in any medium, provided the original work is properly cited.

\begin{abstract}
Aim. The aim of this study was to examine seasonal patterns in glucose tolerance and in the diagnosis of gestational diabetes mellitus (GDM). Methods. Altogether, 11538 women underwent a 75-g oral glucose tolerance test (OGTT) in the twenty-eighth week of pregnancy during the years 2003-2005 in southern Sweden. GDM was defined by the 2-h capillary glucose concentration in the OGTT ( $\geq 8.9 \mathrm{mmol} / \mathrm{L})$. Chi-squared test, analysis of variance, and regression analyses were used for statistical evaluations. Results. The seasonal frequency of GDM ranged from $3.3 \%$ in spring to $5.5 \%$ in summer $(p<0.0001)$. Mean 2 -h glucose concentrations followed the same seasonal trend, with a difference of $0.15 \mathrm{mmol} / \mathrm{L}$ between winter and summer $(p<0.0001)$. The 2-h glucose level increased by $0.009 \mathrm{mmol} / \mathrm{L}$ for every degree increase in temperature $(p<0.0001)$. In regression analysis, summer (June-August) was associated with increased 2-h glucose level $(p<0.001)$ and increased frequency of GDM compared to the other seasons (odds ratio $1.51,95 \%$ confidence interval $1.24-1.83$, and $p<0.001)$. Conclusions. Our findings suggest seasonal variation in the 2 -h glucose concentration in the OGTT and in the proportion of women diagnosed with GDM, with a peak in the summer.
\end{abstract}

\section{Introduction}

While seasonality in the onset of type 1 diabetes is well documented [1], less is known about seasonality in the diagnosis of type 2 and gestational diabetes mellitus (GDM). Doró et al. (2006) reported an increased incidence of type 2 diabetes onset in winter [2]. A similar pattern in HbAlc and glucose levels has been reported in diabetes patients, possibly because of seasonal variations in environmental conditions, such as diet and exercise [3-6]. In contrast, Schmidt et al. (1994) reported a 4-fold increase in the frequency of GDM in the summer compared with the winter, which they related to increased 2-h glucose levels in the oral glucose tolerance test (OGTT) at higher ambient temperatures [7]. Similar results of temperature-induced differences in postload venous glucose levels have previously been reported by Akanji et al. in subjects with and without diabetes $[8,9]$.
However, two other studies from Australia and the UK found no clinically significant evidence of any seasonal variation in the prevalence of GDM or the 2-h glucose levels in the OGTT $[10,11]$.

The prevalence of GDM in a given population depends on the screening approach and on the diagnostic criteria used [12]. In southern Sweden, universal screening for GDM with a 75-g OGTT has been performed at the antenatal clinics since 1995 , with no major changes in the diagnostic procedure. The diagnosis of GDM is based on the 2-h capillary glucose concentration [13]. During the years 2003-2005, pregnant women, representing different glucose categories according to the OGTT, were invited to take part in a follow-up study postpartum, the Mamma Study. During the period of recruitment, a large number of test results were made available and they form the basis of the present study. The aim was to determine whether there were any differences 
in glucose tolerance by season and, consequently, if the frequency of GDM showed any seasonal variation. Such differences are important to elucidate, since they may affect the diagnostic procedure and interpretation of the results.

\section{Materials and Methods}

2.1. GDM Screening. The screening programme for GDM in southern Sweden has been described in detail elsewhere [13]. Briefly, a 75-g OGTT is offered to all women in the twentyeighth week of gestation after overnight fasting and also in week 12 if there is a history of GDM in previous pregnancies or a first-degree relative with diabetes. The HemoCue blood glucose 201+ system (HemoCue AB, Ängelholm, Sweden) is used to perform immediate analysis of capillary glucose concentrations, which are reported as equivalent plasma glucose concentrations [14]. To ascertain the quality of the individual testing, double sampling is used, with acceptance of a divergence of $\leq 0.3 \mathrm{mmol} / \mathrm{L}$. If this is not reached, a third sample is taken, and if the divergence between two of the samples is still not acceptable, the equipment is checked and the OGTT is not regarded as being valid.

The diagnostic criteria for GDM used in the present study are a slight modification of those recommended by the World Health Organization in 1999, defining GDM as a 2 -h venous plasma glucose concentration of $\geq 7.8 \mathrm{mmol} / \mathrm{L}$, corresponding to a capillary plasma glucose concentration of $\geq 8.9 \mathrm{mmol} / \mathrm{L}$ [15]. The diagnosis was based on the mean of the two measurements.

2.2. Study Population. Recruitment to the Mamma Study took place during the years 2003-2005 and involved four of the five delivery departments in the county of Skane in southern Sweden [16]. During the recruitment period, OGTT results from the local antenatal clinics were sent to the study coordinator, enabling identification of the test results of women who consented to be enrolled; it also ensured correct sampling technique [13]. Initially, 11976 OGTT results in total were reported. If a woman had more than one pregnancy during the study period, only the first one was included. Likewise, if a woman underwent more than one OGTT during the same pregnancy, only the one performed in pregnancy week 28 was included. This restricted data set $(n=11538)$ formed the basis of the present evaluation. Mean monthly temperatures during the study period were obtained from the Swedish Meteorological and Hydrological Institute (http://opendata-download-metobs.smhi.se/explore/?parameter=3\#).

The study was carried out in accordance with the Declaration of Helsinki. Written informed consent was obtained from all participants, and the study protocol was approved by the Ethics Committee of Lund University (LU 259-00).

2.3. Statistical Analysis. OGTT results from the 3-year study period were grouped together into months and seasons (winter: December-February; spring: March-May; summer: June-August; autumn: September-November). Chi-squared test was used to test for differences in frequencies between months and seasons, and one-way analysis of variance (ANOVA) was used to test for the corresponding differences in means. Multivariable logistic regression was used to examine whether month or season was associated with the diagnosis of GDM and multivariable linear regression was used to examine the corresponding associations with 2 -h glucose levels. The relationship between mean monthly temperatures and mean monthly 2 -h glucose concentrations was evaluated by simple linear regression.

IBM SPSS Statistics 22 for Windows (IBM Corporation, Armonk, NY) was used for analysis. Two-sided $p$ values of less than 0.05 were considered to be statistically significant.

\section{Results}

Of the 11538 women who underwent an OGTT during the study period, a total of 487 women (4.2\%) were diagnosed with GDM.

Table 1 shows the study material, organized by month and season. The monthly frequency of GDM ranged from $2.9 \%$ in March to $5.8 \%$ in June, and the seasonal frequency of GDM ranged from $3.3 \%$ in spring to $5.5 \%$ in summer. The differences in frequencies were statistically significant, both for month $(p=0.01)$ and for season $(p<0.0001)$. The mean age of participating women was 29.9 (standard deviation 5.1) years, and the ages ranged from 15 to 49 years. The age of the women differed statistically significantly between months and seasons $(p<0.001)$. However, no significant differences in the monthly distributions of age were noted.

Mean monthly temperature ranged from $-0.6^{\circ} \mathrm{C}$ in winter to $17.7^{\circ} \mathrm{C}$ in summer (Table 1 ). In a simple linear regression with 2 -h plasma glucose as the dependent variable and mean monthly temperature as the predictor variable, the coefficient in the equation was 0.009 , suggesting that the 2-h glucose level increased by $0.009 \mathrm{mmol} / \mathrm{L}$ for every degree increase in temperature $(p<0.0001)$.

Figure 1 illustrates the monthly mean 2-h glucose level during the OGTT (with 95\% confidence interval (CI)) and the monthly percentage of women with GDM. Though numerically small, the differences in 2-h glucose levels were statistically significant $(p<0.001)$, with the lowest values observed from January to March and peak levels from June to August. A similar seasonal trend was seen for the percentage of women with 2 -h glucose levels in the GDM range (2-h glucose level $\geq 8.9 \mathrm{mmol} / \mathrm{L}$ ). There were no differences in the distribution of glucose concentrations between months or seasons.

In regression analysis, adjusting for age, the summer months (June to August) were found to be associated with increased 2-h glucose levels $(p<0.001)$ and increased frequency of GDM compared to all other months $(\mathrm{OR}=1.51$ (95\% CI: 1.24-1.83); $p<0.001)$.

\section{Discussion}

In this observational study of 11538 pregnancies, we found seasonal variations in the 2 -h glucose level in the OGTT performed in the twenty-eighth week of gestation, giving 
TABLE 1: Description of the study material, organized by month and season.

\begin{tabular}{|c|c|c|c|c|c|}
\hline & $\begin{array}{c}\text { OGTT } \\
n \\
\end{array}$ & $\begin{array}{l}\text { GDM } \\
n(\%)\end{array}$ & $\begin{array}{c}\text { 2-h glucose, } \mathrm{mmol} / \mathrm{L} \\
\text { Mean (SD) }\end{array}$ & $\begin{array}{l}\text { Age, years, } \\
\text { mean }(\mathrm{SD})\end{array}$ & $\begin{array}{c}\text { Temperature, }{ }^{\circ} \mathrm{C}, \\
\text { mean }\end{array}$ \\
\hline \multicolumn{6}{|l|}{ Month } \\
\hline January & 1094 & $36(3.3)$ & $6.4(1.3)$ & $30.4(5.0)$ & 0 \\
\hline February & 928 & $34(3.7)$ & $6.4(1.3)$ & $30.1(5.3)$ & -0.6 \\
\hline March & 1082 & $31(2.9)$ & $6.4(1.2)$ & $30.1(5.2)$ & 2.4 \\
\hline April & 1027 & $34(3.3)$ & $6.5(1.3)$ & $30.1(5.0)$ & 7.5 \\
\hline May & 1057 & $41(3.9)$ & $6.5(1.4)$ & $30.4(5.1)$ & 12.1 \\
\hline June & 1009 & $59(5.8)$ & $6.6(1.4)$ & $29.8(5.1)$ & 15.2 \\
\hline July & 974 & $50(5.1)$ & $6.6(1.3)$ & $30.0(5.0)$ & 17.7 \\
\hline August & 928 & $52(5.6)$ & $6.6(1.3)$ & $29.6(5.0)$ & 17.6 \\
\hline September & 781 & $33(4.2)$ & $6.5(1.3)$ & $29.5(5.3)$ & 14.4 \\
\hline October & 835 & $42(5.0)$ & $6.6(1.3)$ & $29.6(5.1)$ & 8.4 \\
\hline November & 897 & $38(4.2)$ & $6.6(1.3)$ & $29.6(5.2)$ & 5.3 \\
\hline December & 926 & $37(4.0)$ & $6.5(1.3)$ & $29.8(5.1)$ & 2.9 \\
\hline \multicolumn{6}{|l|}{ Season } \\
\hline Winter & 2948 & 107 (3.6) & $6.4(1.3)$ & $30.1(5.1)$ & 0.7 \\
\hline Spring & 3166 & $106(3.3)$ & $6.5(1.3)$ & $30.2(5.1)$ & 7.3 \\
\hline Summer & 2911 & $161(5.5)$ & $6.6(1.4)$ & $29.8(5.0)$ & 16.8 \\
\hline Autumn & 2513 & $113(4.5)$ & $6.6(1.3)$ & $29.6(5.2)$ & 9.2 \\
\hline
\end{tabular}

GDM, gestational diabetes mellitus; OGTT, oral glucose tolerance test; SD, standard deviation.

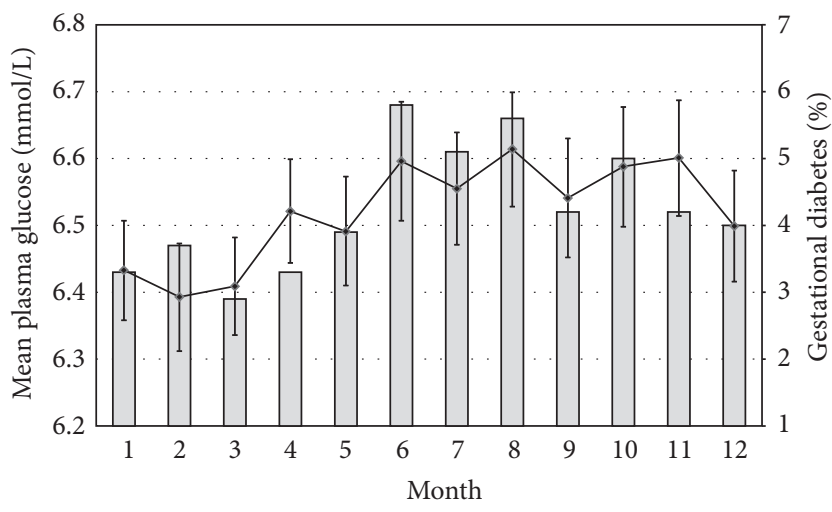

$\square$ Percentage of women with gestational diabetes

- Mean 2-h glucose concentration with 95\% confidence interval

Figure 1: Monthly mean 2-h glucose levels and the monthly percentage of women with gestational diabetes mellitus.

seasonal variations in the percentage of women diagnosed with GDM - with a peak in the summer.

Until recently, there have only been three previous studies in the literature evaluating seasonality in GDM $[7$, $10,11]$. Using a 2 -h venous plasma glucose concentration of $\geq 7.8 \mathrm{mmol} / \mathrm{L}$ to define GDM during a standardized 75 g OGTT in 1113 consecutively tested women in Brazil, Schmidt et al. (1994) reported that the frequency of GDM varied in relation to the ambient temperature, from $4.8 \%$ in winter to $14.8 \%$ in summer [7]. For every degree increase in temperature, the mean 2-h glucose level increased by
$0.07 \mathrm{mmol} / \mathrm{L}$, while the fasting glucose levels were unaffected by temperature. Standardizing the results at $23^{\circ} \mathrm{C}$ indicated that women were overdiagnosed by $19 \%$ at higher temperatures and underdiagnosed by $45 \%$ at lower temperatures [7]. Similar results have been described in small-scale studies outside of pregnancy $[8,9]$. Increased arterialisation of the venous blood at elevated temperatures has been suggested to be a plausible explanation [17]. Whether these variations in glucose levels result from an acute effect of temperature rather than a chronic one is not fully understood, although some experimental studies have indicated an acute effect $[18,19]$. Interestingly, in a very recent study from the coastal area of Australia, Moses et al. reported significantly lower median 1$\mathrm{h}$ and 2-h glucose levels in the OGTT in the winter compared with the overall $1-\mathrm{h}$ and 2 -h results, when evaluated in a cohort of 7369 pregnant women prospectively followed up during a 3-year period [20]. Furthermore, in a populationbased study from South Australia, Verburg et al. recently reported seasonal variation in the diagnosis of GDM based on the estimated date of conception [21].

Since the present study was based on capillary glucose measurements, previous findings cannot be directly extrapolated to results using our methodology. However, temperature-induced changes in peripheral blood flow may very well affect the composition of capillary blood as well, representing a mixture of arterial and venous blood. According to national statistics, the average temperature in the region varied between $-0.6^{\circ} \mathrm{C}$ in winter and $+17.7^{\circ} \mathrm{C}$ in summer during the study period. With respect to the simple linear regression analysis, indicating that for every one degree increase in temperature the glucose concentration 
increased by $0.009 \mathrm{mmol} / \mathrm{L}$, this difference in temperature between summer and winter corresponds to a difference of $0.15 \mathrm{mmol} / \mathrm{L}$ in glucose concentration. It is important to note that OGTT as such has a rather low reproducibility, especially for 2-h glucose levels in the intermediate range [22-24]. This means that a difference of $0.15 \mathrm{mmol} / \mathrm{L}$ would have diagnostic implications in women with glucose concentrations close to the diagnostic limit, thereby increasing the frequency of GDM in the summer.

Though not regarded as a diagnostic standard [25], capillary finger-tip tests have been used in the screening programme for GDM in southern Sweden ever since they were first introduced in 1995 [13]. For practical reasons, this was regarded as a prerequisite when introducing the OGTT on a large scale. Glucose measurements based on capillary samples are generally believed to show greater variation than venous glucose measurements [26]. If the hand is cold there is a risk of squeezing and "milking" of the finger, increasing the proportion of extracellular fluid, resulting in a lower glucose concentration. However, since glucose measurements in the present study were based on measurements after two hours at room temperature in the antenatal clinic, such an explanation would seem less likely.

In contrast to our findings and those of Schmidt et al. [7], Janghorbany et al. (2006) could not prove any seasonality in glucose tolerance or in the incidence of GDM in 4942 pregnancies in Plymouth, UK [11]. However, it is important to note that Plymouth has a very mild climate, with little seasonal variation. Furthermore, only $11 \%(n=539)$ of the women underwent an OGTT during pregnancy and the incidence figures were based on those with an abnormal OGTT ( $n=90)$, as opposed to all the others who either had a normal OGTT or initially screened negative by random plasma glucose testing or risk factors. In a study from Australia, Moses and Griffiths (1995) examined seasonal trends in 2749 women consecutively tested with a 75-g OGTT [10]. Interestingly, after adjustments for various risk factors, multiple regression analysis revealed a significant association between the monthly temperature and the 2-h glucose level in the OGTT; the 2 -h glucose level increased by $0.026 \mathrm{mmol} / \mathrm{L}$ for every degree increase in temperature [10]. However, Chisquared analysis did not suggest any seasonal variation in the diagnosis of GDM, and it was concluded that the association between 2-h glucose and temperature was unlikely to be of any clinical importance in the temperate coastal area of Australia, with the mean monthly temperature during the study period ranging from $13.6^{\circ} \mathrm{C}$ in July to $22.3^{\circ} \mathrm{C}$ in January [10].

Our results do not agree with previous observations by Doró et al. from Hungary, of an increased incidence of type 2 diabetes in winter, although it should be noted that the incidence figures reported were based on the initiation of antidiabetic therapy in individuals with previously diagnosed diabetes, therefore not representing the "true" diabetes onset [2]. Likewise, worsening of metabolic control in subjects with type 2 diabetes in winter has been described in a number of studies [3-6]. Since diet and exercise are hallmarks of the treatment of type 2 diabetes, it is reasonable to assume that environmental factors, such as diet and exercise patterns, have an important role in the seasonal variation in glucose metabolism in patients with diabetes. Seasonal variation in the diagnosis of GDM possibly reflects seasonality of environmental influences early in gestation, during placental development, affecting placental metabolism and glucose homeostasis later on in pregnancy [21]. Many factors vary with season, including the nutritional quality of foods, temperature, the number of hours of sunshine, and vitamin $\mathrm{D}$ synthesis. Maternal vitamin $\mathrm{D}$ deficiency in early pregnancy has been associated with increased risk of GDM [27]. Moreover, seasonal variation in vitamin D status, quantified as the total number of hours of sunshine during the three months preceding the onset of diabetes, was suggested as an explanation for the seasonality of type 2 diabetes reported by Doró et al. [28].

The strength of the present study was the uniform diagnostic procedure for GDM, based on universal screening with a 75-g OGTT and enabling identification of a large cohort of women in one particular week of gestation. Unfortunately, we did not have access to individual data other than age, so the figures are crude and unadjusted for other potentially important risk factors, such as body mass index and ethnicity [29]. However, we have no reason to believe that this would have any major effect on the results, due to the size of the material and the exclusive use of OGTT. Another weakness of the study was that we only had information on mean monthly temperatures during the study period and not the mean temperature on specific days, which makes it difficult to draw any firm conclusions on the effect of temperature on our results.

We have previously shown that approximately 5-6\% of women with GDM in our region have positive islet cell autoantibodies as a marker of autoimmune pathogenesis and therefore of type 1 diabetes [30,31]. Of these, $50 \%$ had developed type 1 diabetes within ten years postpartum [30]. Seasonality in the incidence of type 1 diabetes has been described [1], but this is probably not of any relevance to the present findings, due to the overall low proportion of women who would be expected to have autoimmune diabetes in our material.

\section{Conclusions}

Based on a universally performed OGTT in the twentyeighth week of pregnancy, seasonality in the proportion of women diagnosed with GDM was observed, with a peak in the summer. The mean 2 -h glucose concentration in the OGTT followed the same seasonal trend. The findings may be related to the increased ambient temperature in the summer. Further studies are needed to determine whether our results are reproducible and if they are, to investigate the cause(s) of seasonality, as such variations may have implications for the diagnostic procedure and for interpretation of the results.

\section{Disclosure}

An earlier version of this work was presented as an abstract at 2016 European Association for the Study of Diabetes (EASD) annual conference in Munich. 


\section{Competing Interests}

The authors declare that they have no competing interests.

\section{Authors' Contributions}

Kerstin Berntorp conceived the study. All the authors contributed to the study design and interpretation of data. Kerstin Berntorp wrote the initial draft of the manuscript, and Anastasia Katsarou and Rickard Claesson contributed equally to the subsequent draft. Claes Ignell helped analyse data. Claes Ignell and Nael Shaat critically reviewed and edited the manuscript. All the authors read and approved the final manuscript.

\section{Acknowledgments}

The authors thank Eva Anderberg, who coordinated the Mamma Study and collected the OGTT data for this study. They are indebted to Helene Jacobsson, biostatistician at the Skåne R\&D Centre, University Hospital, Lund, Sweden, for statistical support. The study was supported by grants from the Research Funds of Skåne University Hospital and from the Skåne County Council Research and Development Foundation.

\section{References}

[1] C. C. Patterson, E. Gyürüs, J. Rosenbauer et al., "Seasonal variation in month of diagnosis in children with type 1 diabetes registered in 23 European centers during 1989-2008: little shortterm influence of sunshine hours or average temperature," Pediatric Diabetes, vol. 16, no. 8, pp. 573-580, 2015.

[2] P. Doró, R. Benko, M. Matuz, and G. Soós, "Seasonality in the incidence of type 2 diabetes: a population-based study," Diabetes Care, vol. 29, no. 1, p. 173, 2006.

[3] W. W. Liang, "Seasonal changes in preprandial glucose, A1C, and blood pressure in diabetic patients," Diabetes Care, vol. 30, no. 10, pp. 2501-2502, 2007.

[4] A. Kershenbaum, A. Kershenbaum, J. Tarabeia, N. Stein, I. Lavi, and G. Rennert, "Unraveling seasonality in population averages: an examination of seasonal variation in glucose levels in diabetes patients using a large population-based data set," Chronobiology International, vol. 28, no. 4, pp. 352-360, 2011.

[5] C.-L. Tseng, M. Brimacombe, M. Xie et al., "Seasonal patterns in monthly hemoglobin Alc values," American Journal of Epidemiology, vol. 161, no. 6, pp. 565-574, 2005.

[6] M. Sohmiya, I. Kanazawa, and Y. Kato, "Seasonal changes in body composition and blood HbAlc levels without weight change in male patients with type 2 diabetes treated with insulin," Diabetes Care, vol. 27, no. 5, pp. 1238-1239, 2004.

[7] M. I. Schmidt, M. C. Matos, L. Branchtein et al., "Variation in glucose tolerance with ambient temperature," The Lancet, vol. 344, no. 8929, pp. 1054-1055, 1994.

[8] A. O. Akanji and R. N. Oputa, "The effect of ambient temperature on glucose tolerance," Diabetic Medicine, vol. 8, no. 10, pp. 946-948, 1991.

[9] A. O. Akanji, M. Bruce, K. Frayn, T. D. R. Hockaday, and G. M. Kaddaha, "Oral glucose tolerance and ambient temperature in non-diabetic subjects," Diabetologia, vol. 30, no. 6, pp. 431-433, 1987.

[10] R. Moses and R. Griffiths, "Is there a seasonal variation in the incidence of gestational diabetes?" Diabetic Medicine, vol. 12, no. 7, pp. 563-565, 1995.

[11] M. Janghorbani, E. Stenhouse, R. B. Jones, and A. Millward, "Gestational diabetes mellitus in Plymouth, U.K.: prevalence, seasonal variation and associated factors," Journal of Reproductive Medicine, vol. 51, no. 2, pp. 128-134, 2006.

[12] K. J. Hunt and K. L. Schuller, "The increasing prevalence of diabetes in pregnancy," Obstetrics and Gynecology Clinics of North America, vol. 34, no. 2, pp. 173-199, 2007.

[13] E. Anderberg, K. Källén, K. Berntorp, A. Frid, and A. Åberg, "A simplified oral glucose tolerance test in pregnancy: compliance and results," Acta Obstetricia et Gynecologica Scandinavica, vol. 86, no. 12, pp. 1432-1436, 2007.

[14] R. W. Burnett, P. D’Orazio, N. Fogh-Andersen et al., "IFCC recommendation on reporting results for blood glucose," Clinica Chimica Acta, vol. 307, no. 1-2, pp. 205-209, 2001.

[15] World Health Organization, Definition, Diagnosis and Classification of Diabetes Mellitus and its Complications. Report of a WHO Consultation. Part 1: Diagnosis and Classification of Diabetes Mellitus, World Health Organization, Geneva, Switzerland, 1999.

[16] K. Berntorp, E. Anderberg, R. Claesson, C. Ignell, and K. Källén, "The relative importance of maternal body mass index and glucose levels for prediction of large-for-gestational-age births," BMC Pregnancy and Childbirth, vol. 15, article 280, 2015.

[17] K. N. Frayn, P. L. Whyte, H. A. Benson, D. J. Earl, and H. A. Smith, "Changes in forearm blood flow at elevated ambient temperature and their role in the apparent impairment of glucose tolerance," Clinical Science, vol. 76, no. 3, pp. 323-328, 1989.

[18] E. A. H. McGuire, J. H. Helderman, J. D. Tobin, R. Andres, and M. Berman, "Effects of arterial versus venous sampling on analysis of glucose kinetics in man," Journal of Applied Physiology, vol. 41, no. 4, pp. 565-573, 1976.

[19] R. G. Moses, M. J. Patterson, J. M. Regan, R. Chaunchaiyakul, N. A. S. Taylor, and A. B. Jenkins, "A non-linear effect of ambient temperature on apparent glucose tolerance," Diabetes Research and Clinical Practice, vol. 36, no. 1, pp. 35-40, 1997.

[20] R. G. Moses, V. C. K. Wong, K. Lambert, G. J. Morris, and F. San Gil, "Seasonal changes in the prevalence of gestational diabetes mellitus," Diabetes Care, vol. 39, no. 7, pp. 1218-1221, 2016.

[21] P. E. Verburg, G. Tucker, W. Scheil, J. J. Erwich, G. A. Dekker, and C. T. Roberts, "Seasonality of gestational diabetes mellitus: a South Australian Population Study," BMJ Open Diabetes Research \& Care, vol. 4, no. 1, Article ID e000286, 2016.

[22] K. Schousboe, J. E. Henriksen, K. O. Kyvik, T. I. A. Sørensen, and P. Hyltoft Petersen, "Reproducibility of S-insulin and Bglucose responses in two identical oral glucose tolerance tests," Scandinavian Journal of Clinical and Laboratory Investigation, vol. 62, no. 8, pp. 623-630, 2002.

[23] C. M. Balion, P. S. Raina, H. C. Gerstein et al., "Reproducibility of impaired glucose tolerance (IGT) and impaired fasting glucose (IFG) classification: a systematic review," Clinical Chemistry and Laboratory Medicine, vol. 45, no. 9, pp. 1180-1185, 2007.

[24] J. M. Mooy, P. A. Grootenhuis, H. De Vries et al., "Intraindividual variation of glucose, specific insulin and proinsulin concentrations measured by two oral glucose tolerance tests in a general Caucasian population: the Hoorn Study, Diabetologia, vol. 39, no. 3, pp. 298-305, 1996. 
[25] World Health Organization and International Diabetes Federation, Definition and Diagnosis of Diabetes Mellitus and Intermediate Hyperglycaemia: A Report of a WHO/IDF Consultation, World Health Organization, Geneva, Switzerland, 2006.

[26] B. Carstensen, J. Lindström, J. Sundvall, K. Borch-Johnsen, and J. Tuomilehto, "Measurement of blood glucose: comparison between different types of specimens," Annals of Clinical Biochemistry, vol. 45, no. 2, pp. 140-148, 2008.

[27] C. Zhang, C. Qiu, F. B. Hu et al., "Maternal plasma 25-hydroxyvitamin D concentrations and the risk for gestational diabetes mellitus," PLOS ONE, vol. 3, no. 11, Article ID e3753, 2008.

[28] P. Doró, W. B. Grant, R. Benko, M. Matuz, T. Tóth, and G. Soós, "Vitamin D and the seasonality of type 2 diabetes," Medical Hypotheses, vol. 71, no. 2, pp. 317-318, 2008.

[29] A. Dornhorst and M. Roosi, "Risk and prevention of type 2 diabetes in women with gestational diabetes," Diabetes Care, vol. 21, supplement 2, pp. B43-B49, 1998.

[30] C. Nilsson, D. Ursing, C. Törn, A. Åberg, and M. LandinOlsson, "Presence of GAD antibodies during gestational diabetes mellitus predicts type 1 diabetes," Diabetes Care, vol. 30, no. 8, pp. 1968-1971, 2007.

[31] A. Papadopoulou, K. F. Lynch, E. Anderberg et al., "HLADQB1 genotypes and islet cell autoantibodies against GAD65 and IA-2 in relation to development of diabetes post partum in women with gestational diabetes mellitus," Diabetes Research and Clinical Practice, vol. 95, no. 2, pp. 260-264, 2012. 


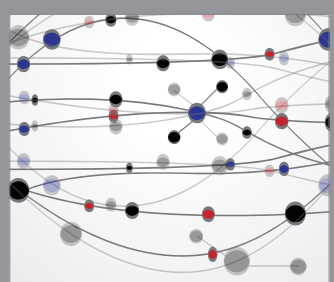

The Scientific World Journal
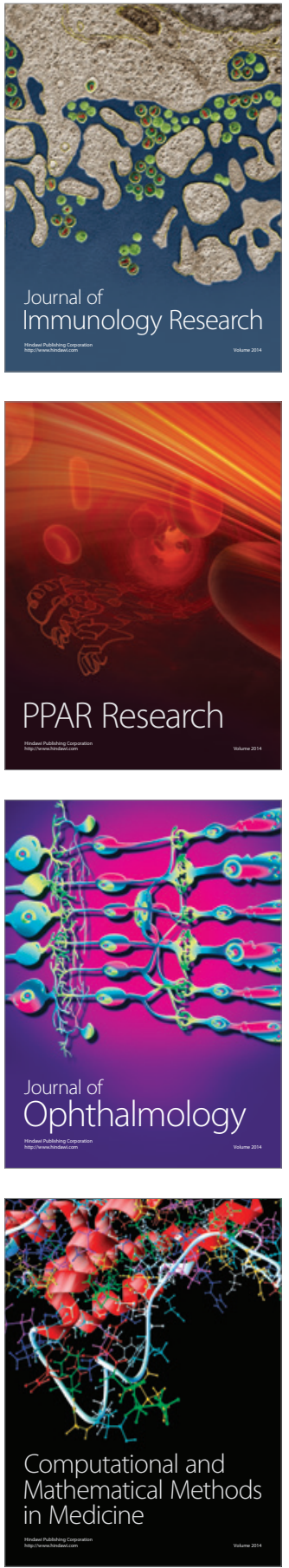

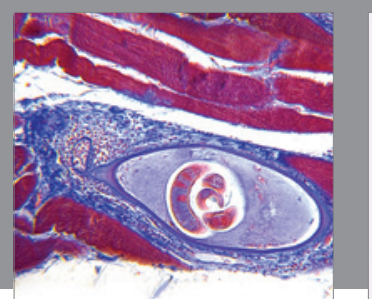

Gastroenterology Research and Practice

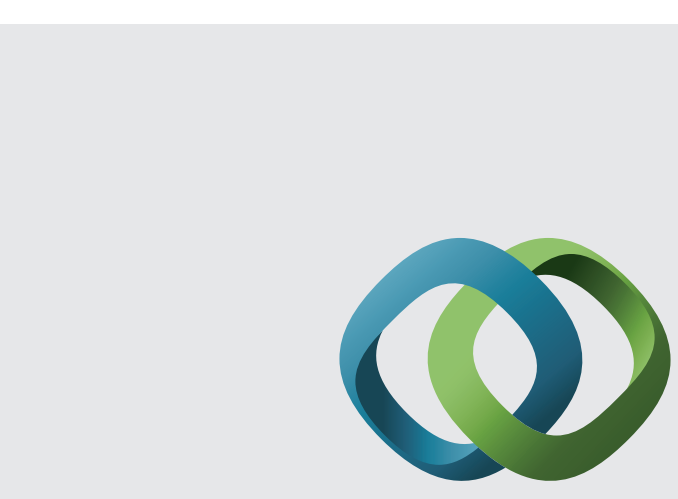

\section{Hindawi}

Submit your manuscripts at

http://www.hindawi.com
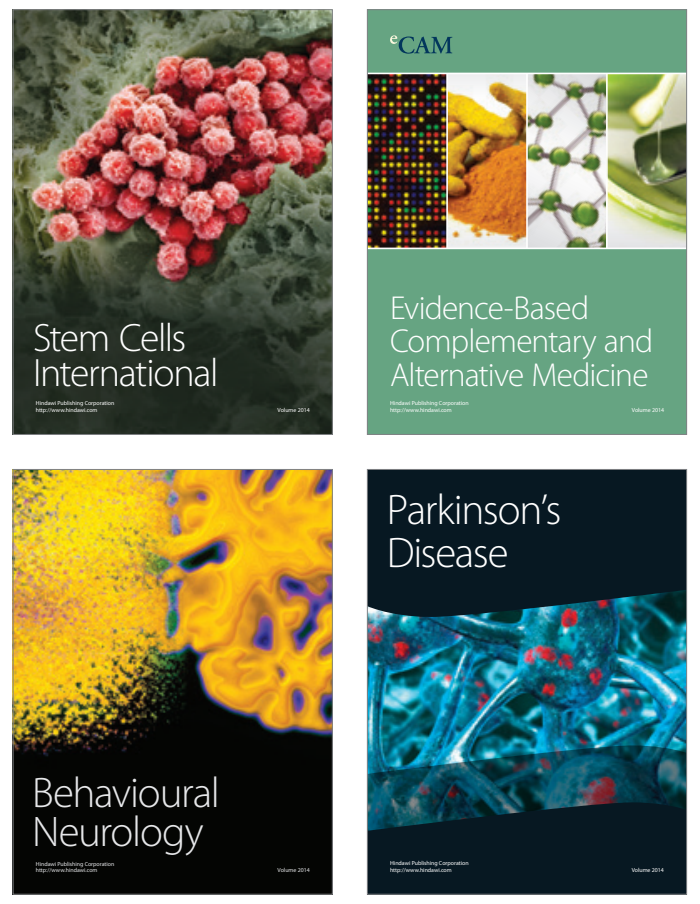
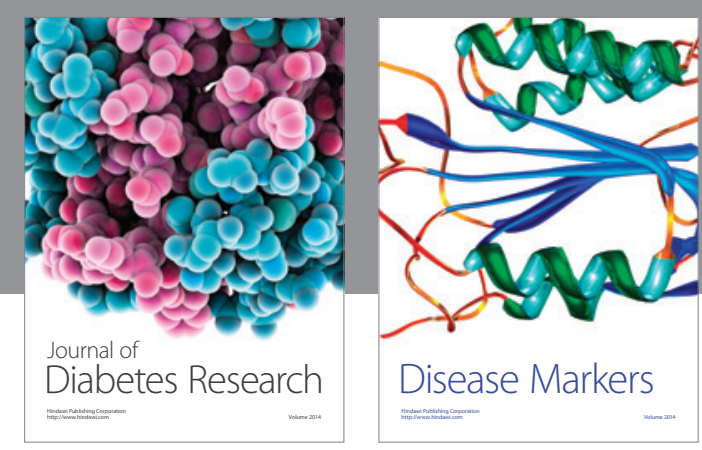

Disease Markers
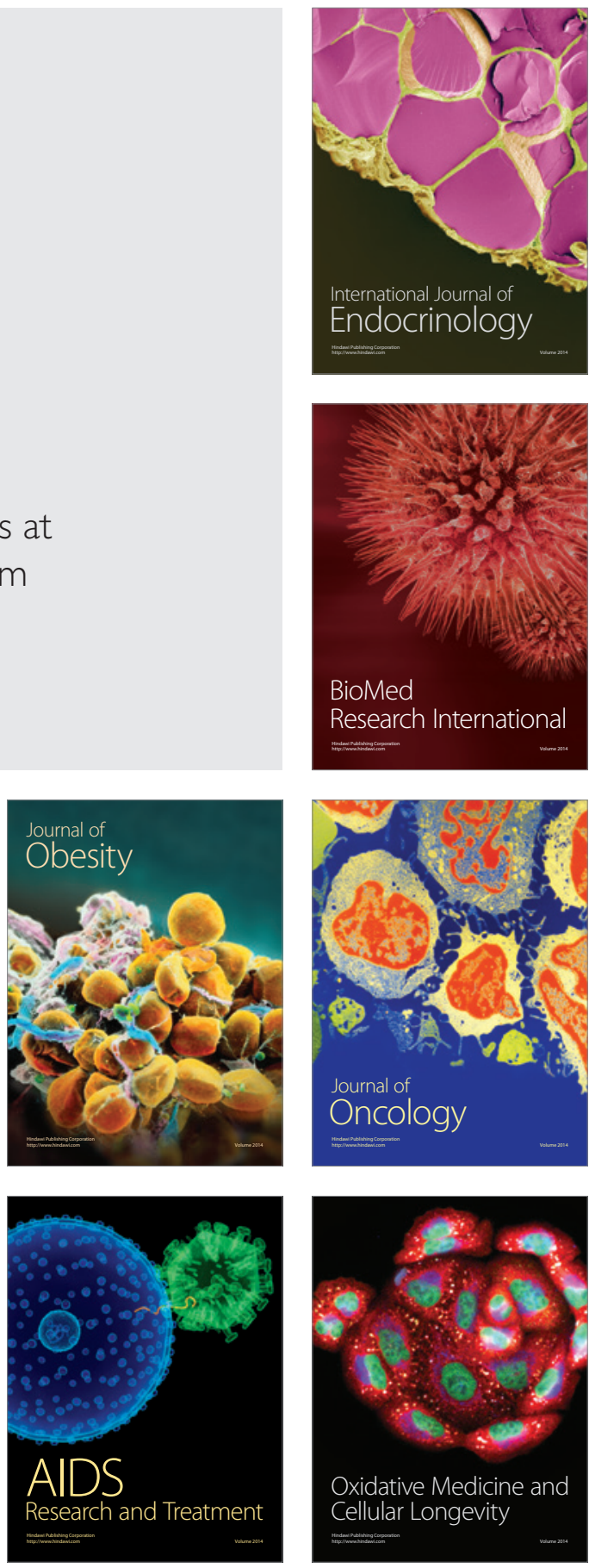